\title{
Genetic Regulation of Mammalian Diversity
}

\author{
R.R. Behringer, ${ }^{1}$ J.J. RAsweiler IV, ${ }^{2}$ C.-H. Chen, ${ }^{1}$ AND C.J. CRetekos ${ }^{3}$ \\ ${ }^{1}$ Department of Genetics, University of Texas M.D. Anderson Cancer Center, Houston, Texas 77030; \\ ${ }^{2}$ Department of Obstetrics and Gynecology, State University of New York Downstate Medical Center, Brooklyn,
} New York 11203; ${ }^{3}$ Department of Biological Sciences, Idaho State University, Pocatello, Idaho 83209

Correspondence: rrb@mdanderson.org

\begin{abstract}
Mammals have evolved a variety of morphological adaptations that have allowed them to compete in their natural environments. The developmental genetic basis of this morphological diversity remains largely unknown. Bats are mammals that have the unique ability of powered flight. We have examined the molecular embryology of bats and investigated the developmental genetic basis for their highly derived limbs used for flight. Initially, we developed an embryo staging system for a model chiropteran, Carollia perspicillata, the short-tailed fruit bat that has subsequently been used for staging other bat species. Expression studies focusing on genes that regulate limb development indicate that there are similarities and differences between bats and mice. To determine the consequences of these expression differences, we have conducted an enhancer switch assay by gene targeting in mouse embryonic stem cells to create mice whose genes are regulated by bat sequences. Our studies indicate that cis-regulatory elements contribute to the morphological differences that have evolved among mammalian species.
\end{abstract}

The approximately 4800 extant species of the class Mammalia exhibit remarkable morphological and physiological diversity (Behringer et al. 2006). Most of these live on land, whereas others are born and live their entire lives swimming in aquatic environments or are capable of powered flight exploiting aerial niches. We are interested in the developmental genetic basis of the morphological and physiological variation observed among mammalian species.

Mammals are divided into three subclasses: the oviparous (egg-laying) monotremes, the viviparous (livebearing) marsupials, and the eutherians. Most mammalian species are contained in the eutherian subclass, with a majority belonging to two orders: the Rodentia ( $\sim 2000$ species) and the Chiroptera or bats ( 1200 species). We have focused our developmental studies on one of the bats, while also exploiting some of the numerous advantages of the laboratory mouse model.

The chiropterans have traditionally been divided into the megachiroptera and microchiroptera, i.e., simplistically, big bats and small bats. However, the biggest microchiropteran is larger than the smallest megachiropteran. Bats are widely dispersed throughout the world except at the poles. They feed on a wide variety of food sources, including pollen, nectar, fruits, other plant parts (flowers, leaves), insects, spiders, blood, and vertebrates. Many species have the ability of echolocation, using sound to "visualize" their environments and locate food. Among the mammals, bats have evolved the unique ability of powered flight that relies on highly modified limbs. This ability is distinct from other mammals such as flying squirrels (subfamily Petauristinae) and flying lemurs (order Dermoptera) that glide rather than actively sustain flight. The evolution of flight by bats allowed them to exploit a unique niche, the night sky.

The primary model we have chosen for most of our studies is a microchiropteran, Carollia perspillata (the shorttailed fruit bat). Carollia possesses many useful attributes as a model organism for studies of the Chiroptera. It is probably the most abundant mammal inhabiting forested areas in the lowland tropics of the New World and frequently establishes colonies in a variety of man-made structures, from which animals may be easily collected with hand nets. Carollia also readily adapts to captivity in a research setting. Indeed, it is feasible to accomplish this with no or negligible mortalities from the time of initial capture. In captivity, these animals are maintained in modest-sized cages that permit flight, but still facilitate efficient and noninjurious capture for experimental purposes. They are fed a fruit-based diet readily prepared from inexpensive canned and powdered components (Rasweiler et al. 2009). Because Carollia breeds very successfully in captivity with this husbandry program, self-sustaining colonies can be easily established. Finally, pregnancies can be conveniently timed from the first appearance of spermatozoa in daily vaginal smears. This has permitted the accumulation of substantial information on the timing of reproductive and developmental events in captive-maintained animals (Badwaik et al. 1997; Rasweiler et al. 2000; Cretekos et al. 2005). Using embryos derived from timed matings from our laboratory colony, we generated a standardized embryo staging system for Carollia (Cretekos et al. 2005), which has subsequently been modified for use with other species of bats (Giannini et al. 2006; Tokita 2006; Hockman et al. 2009; Nolte et al. 2009). These various factors have enabled our studies on the genetic basis of limb diversity among mammals.

\section{LIMB DIVERSITY AND DEVELOPMENT BETWEEN MICE AND BATS}

Comparisons of the forelimbs of adult mice and bats show significant differences in morphology (Fig. 1). The upper and lower arms of the mouse are approximately 


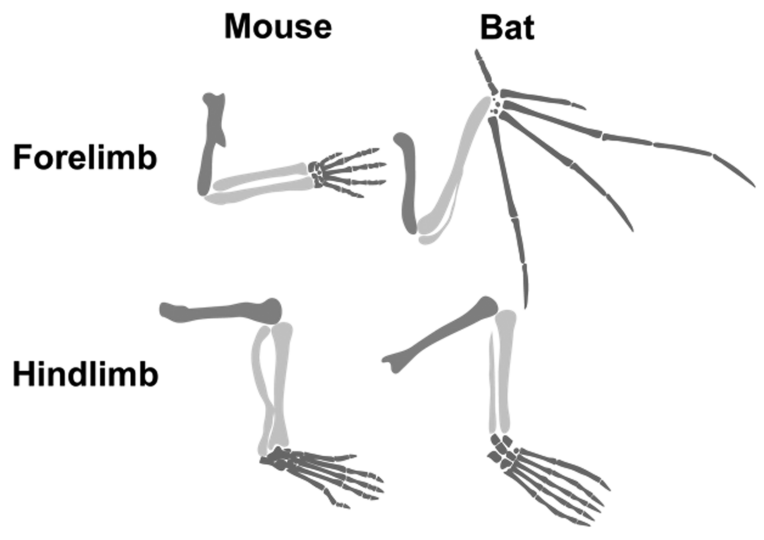

FIGURE 1. Limb comparisons between mouse and bat. Diagram of the limb skeletal elements of the mouse and bat.

equal in length, whereas in the bat the lower arm is about twice as long as the upper arm. The mouse has digits that are separated due to programmed cell death of interdigital tissue during embryogenesis. In the bat, although the first digit of the forelimb is free of interdigital tissue, the four posterior digits (II-V) are connected by the wing membrane. In addition, digits II-V are highly elongated. In contrast, the relative lengths of the segments of the hindlimbs of the mouse and bat are similar. Another batspecific feature includes a significant reduction of the ulna relative to that of the mouse. These morphological adaptations promote the quadrapedal and volant lifestyles of the mouse and bat, respectively.

Perhaps not surprisingly, the embryonic staging systems for all bat species examined so far are very similar in terms of the relative timing, pattern, and initial appearance of major anatomical features (Giannini et al. 2006; Tokita 2006; Hockman et al. 2009; Nolte et al. 2009). Species-spe- cific differences in the shapes and sizes of some structures, such as craniofacial structures, limbs, and tail, appear after initial morphogenesis. In particular, limb outgrowth and early morphogenesis appear to be highly conserved among all bat species examined. Differences in wing proportions corresponding to different flight behaviors of the various species appear during the last third of development (Giannini et al. 2006; Tokita 2006; Hockman et al. 2009; Nolte et al. 2009). For example, digits II-V are spaced widely in the anteroposterior axis, with abundant wing membrane between them in $C$. perspicillata and Pipistrellus abramus (Cretekos et al. 2005; Tokita 2006), whereas digits II-IV are relatively closer together, with less interdigital tissue in Molossus rufus forelimbs, reflecting the relatively broad wing shape in the former species and the narrower wing shape of M. rufus (Nolte et al. 2009).

When limb morphogenesis is compared between bats and mice, a similar progression from early similarity to later differences is observed (Fig. 2). Limb buds form in both mice and bats at a similar stage of development and are morphologically similar in appearance during the early bud stage. Shortly after the formation of the forelimb handplate, the first overt differences appear, with the bat developing a proportionally larger handplate that is posteriorly expanded relative to the symmetric mouse handplate. During subsequent stages, programmed cell death occurs among all of the digits in the mouse, whereas only the interdigital tissue between digits I and II is lost in bat. The remaining interdigital tissue persists and later develops into the chiropatagium or "handwing" of the bat. Finally, during later stages of development, the bat forelimb shows a distinct proximal-to-distal bias in skeletal elongation, whereas the mouse limb skeleton elongates in a relatively uniform proximodistal pattern.

Formation of the axial skeleton begins during Carollia stage (CS) 14, with alcian-blue-positive cartilaginous condensations forming in the midline just ventral to the

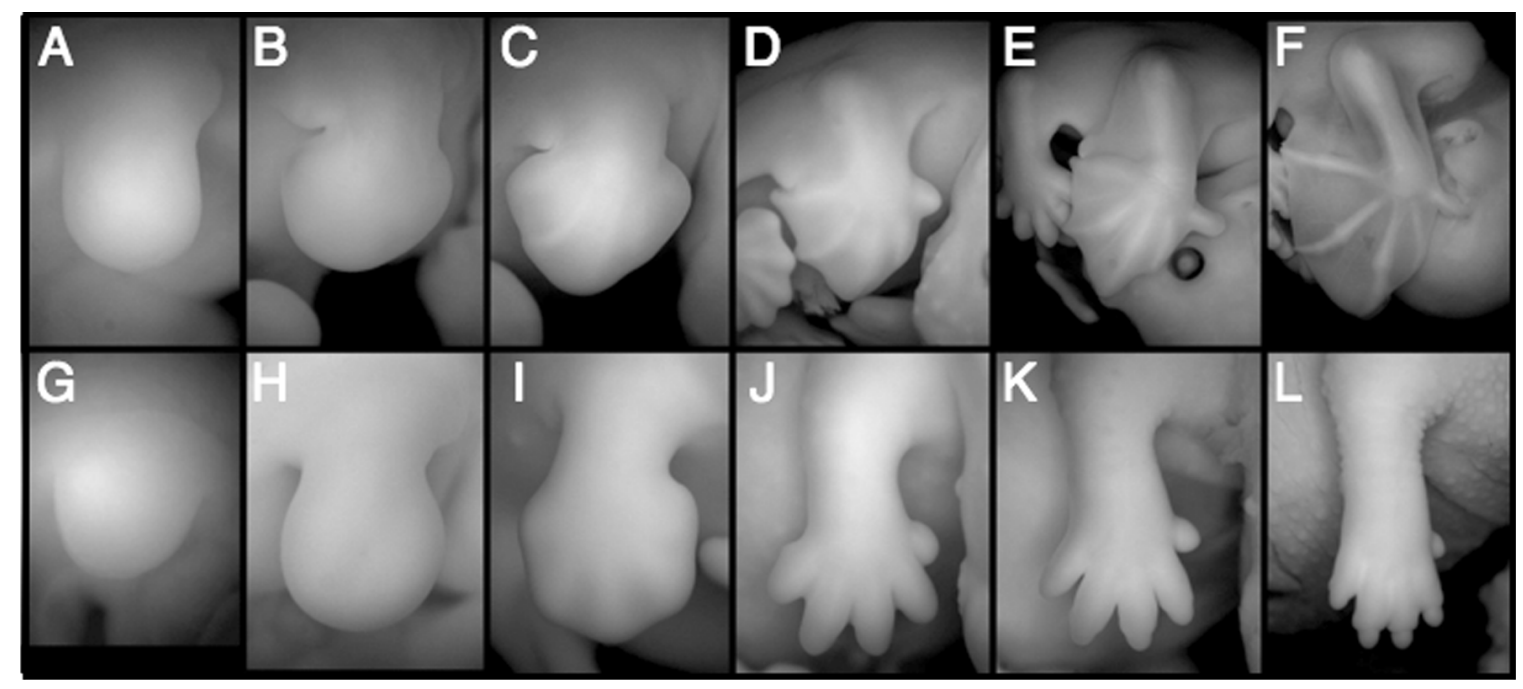

FIGURE 2. Limb development in the bat and mouse. Whole-mount images of forelimbs from bat $(A-F)$ and mouse $(G-L)$. Carollia stages $14(A), 15 \mathrm{E}(B), 15(C), 16(D), 16 \mathrm{~L}(E), 19(F)$; mouse stages $10 \mathrm{dpc}(G), 11 \mathrm{dpc}(H), 12 \mathrm{dpc}(I), 13 \mathrm{dpc}(J), 14 \mathrm{dpc}(K), 16$ $\mathrm{dpc}(L)$. Anterior is to the right. (Reprinted, with permission, from Cretekos et al. 2008.) 
spinal cord (Fig. 3). During subsequent stages, the axial skeleton elaborates, with neural arches extending dorsally from the vertebral bodies by late-stage CS 15 and closing dorsally over the spinal cord by early-stage CS 17 and ribs extending ventrally beginning during CS 16 . The first indication of the appendicular skeleton appears as an alcian-blue-positive condensation in the proximal forelimb during CS 14 (Fig. 3). During subsequent stages, the cartilaginous anlagen of each of the adult appendicular skeletal elements condense sequentially from proximal to distal (Hockman et al. 2008). In the forelimb, the scapula

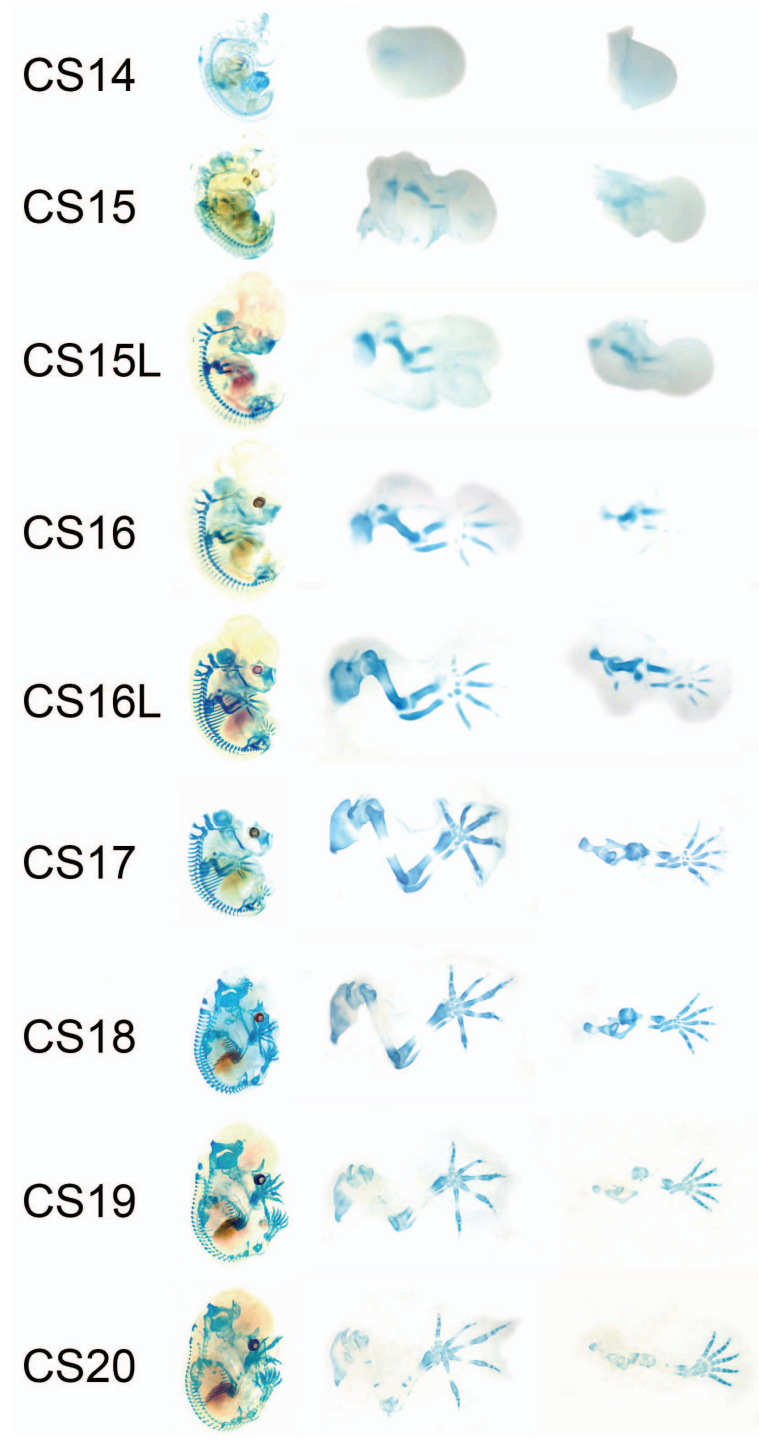

FIGURE 3. Development of the cartilaginous skeleton of Carollia perspicillatta. Whole-mount alcian blue staining of bat embryos from CS 14 through CS 20 . The first column shows lateral views with anterior to the top and dorsal to the left. The second column shows high-magnification views of dissected forelimbs shown with anterior to the top and proximal to the left. The third column shows high-magnification views of dissected hindlimbs shown with anterior to the top and proximal to the left. Embryos are staged according to Cretekos et al. (2005) and indicated for each row. Views are not to scale. and humerus precursors appear during late-stage CS 14, followed by radius and ulna during early-stage CS 15 , carpals and metacarpals by late-stage CS 15, and phalanges during CS 16. During subsequent stages, the alcian-blue-staining cartilages are gradually replaced by ossified bone, again with a proximal-to-distal temporal bias, starting in the central region of each element and spreading with time toward the ends. Hindlimb skeletogenesis, consistent with the timing of limb bud formation, begins about one stage later than that of the forelimb, but catches up by CS 17 (Fig. 3).

Analysis of the cartilaginous skeleton of a stage-17 Carollia embryo reveals that the forming skeletal elements are relatively similar to the mouse, i.e., the upper and lower arm bones are of approximately equal length and each of the five digits are also approximately equal in length (Fig. 4A). However, by the time of birth, the forelimb skeletal elements have taken on the bat-specific morphological characters of the adult, including bone lengthening and ulna reduction (Fig. 4B). These findings suggest that limb form initially follows a general vertebrate pattern of development but, at later stages of gestation, species-specific characters emerge.

\section{GENE EXPRESSION DURING BAT LIMB DEVELOPMENT}

Many genes have been identified, predominantly in the chick and mouse systems, that are expressed in unique temporal and spatial patterns during vertebrate limb development. Their functions in limb patterning and morphogenesis have been determined for a subset of these genes. The expression of some of these genes have also been determined during bat limb development, including Hoxd13 (Chen et al. 2005; Ray and Capecchi 2008), Fgf8 (Weatherbee et al. 2006; Cretekos et al. 2007), Bmp2, Bmp4, Bmp7, Msx2, Gremlin, and Spry2 (Weatherbee et al. 2006), phospho-Smad1/5/8 (Sears et al. 2006), Prx1 (Cretekos et al. 2008), and Shh and Ptcl (Hockman et al. 2008). The general findings from these studies are that the expression patterns of orthologous genes in the mouse
A

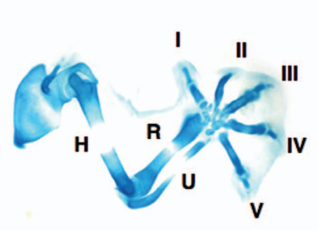

Stage 17 (56 dpc)
B

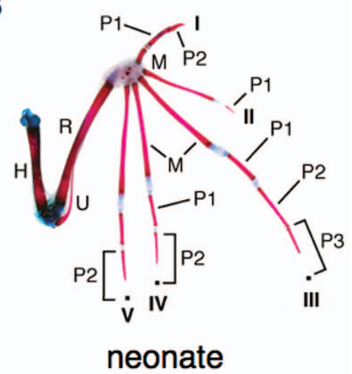

FIGURE 4. Bat-specific characters develop late in gestation. Carollia forelimb skeletons at CS $17(A)$ and newborn $(B)$. $(A)$ Alcian blue preparation, $(B)$ alcian blue/alizarin red preparation. Digits are numbered I-V. (dpc) Days postcoitus, $(\mathrm{H})$ humerus, (M) metacarpel, (P) phalange, $(\mathrm{R})$ radius, $(\mathrm{U})$ ulna. (B, Reprinted, with permission, from Chen et al. 2005 [C Wiley].) 
and bat are generally similar, but many times, speciesspecific differences occur notably at later stages of embryogenesis. For example, Hoxd13 is expressed in the posterior region of the developing mouse limb bud. Although Hoxd13 expression in the bat forelimb is initially similar to that of the mouse, at later stages the anterior boundary of expression is shifted to a more posterior position relative to mouse Hoxd13 (Chen et al. 2005). Likewise, Fgf8 expression is detected in the apical ectodermal ridge (AER) of the forming mouse and bat limb buds. In the bat, the width of $F g f 8$ AER expression is significantly greater than that of the mouse (Cretekos et al. 2007). These expression studies provided a foundation for assaying the function of bat genes in the mouse.

\section{PRX1}

Prx1 (also called MHox or Prrx1) encodes a pairedclass homeodomain transcription factor. The mouse locus produces two isoforms by alternative splicing, encoding proteins that differ only in the carboxy-terminal region. Alignment of the deduced amino acid sequences of Carollia PRX1 isoforms shows more than $99 \%$ identity with the orthologous isoforms of mouse PRX1 (Cretekos et al. 2008). Only two amino acid differences are found between bat and mouse PRX1 proteins, and these occur outside of the conserved homeodomain, PRX and OAR motifs, suggesting that the orthologs have identical biochemical activities.

Prx 1 is expressed in a complex and dynamic pattern in the limb from the early undifferentiated bud stage through outgrowth and morphogenesis, suggesting roles in multiple steps of limb development (Fig. 5A) (Cserjesi et al. 1992; Martin et al. 1995). Expression of Prxl in the developing bat limb shows a striking up-regulation in the distal limb compared to mouse (Cretekos et al. 2008). Analysis of the mouse Prxl knockout (null) phenotype demon-

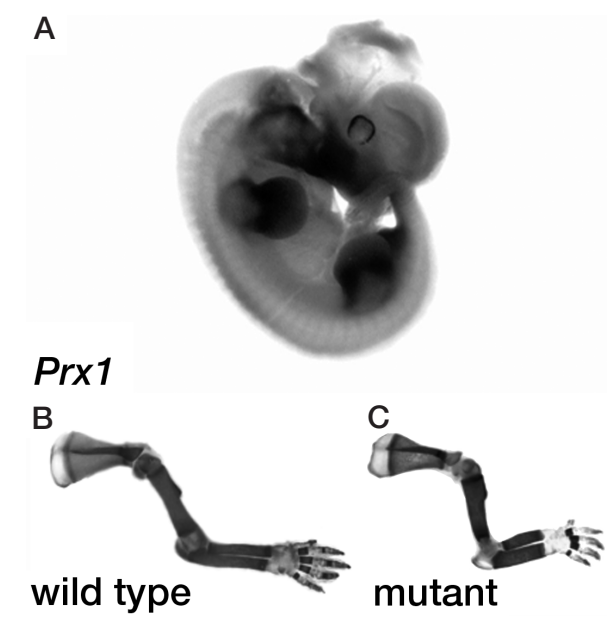

FIGURE 5. $\operatorname{Pr} x 1$ expression and mutant forelimb phenotype in the mouse. (A) Prxl whole-mount in situ hybridization of 11.5dpc mouse embryo. Expression is detected predominantly in the craniofacial region and limbs. $(B, C)$ Alcian blue/alizarin red forelimb skeleton preparations from wild-type $(B)$ and $\operatorname{Pr} x 1^{-1-}$ (C) newborn mice. strates an essential role in the morphogenesis of limb and craniofacial tissues. The limbs of mice with Prxl function eliminated display shortened zeugopod (forearm and lower leg) elements and numerous autopod (hand and foot) element reductions and malformations (Fig. 5B) (Martin et al. 1995; ten Berge et al. 1998; Lu et al. 1999).

A region in which sequences capable of regulating expression in the limb reside, just upstream of the start of transcription, had been previously identified (Martin and Olson 2000). We identified the bat ortholog of the Prxl limb-specific enhancer by virtue of its conserved sequence and genomic position relative to mouse and human orthologs, and characterized the activity of this enhancer by combining it with a basal promoter and lac $Z$ reporter in transgenic mice. The Carollia Prxl limb enhancer shares $\sim 80 \%$ sequence identity with that of mouse and directs the expression of the reporter in a pattern similar to that of the orthologous mouse enhancer in the limbs of transgenic mice (Cretekos et al. 2008).

\section{ENHANCER SWITCH AND DELETION BY GENE TARGETING}

The challenge in studying the evolution of gene regulation is to distinguish between unselected genetic drift and significant functional divergence in noncoding cis-regulatory sequences (Cretekos et al. 2001). To address this challenge, we replaced the mouse Prxl limb enhancer with the bat limb enhancer within the endogenous locus by gene targeting in mouse embryonic stem (ES) cells and generated mice from these ES cells $\left(\operatorname{Pr} x 1^{\text {BatE }}\right)$ (Cretekos et al. 2008). We replaced the limb enhancer sequences without altering the coding sequence; thus, any change in Prx function in the $\operatorname{Prx} 1^{\text {BatE }}$ mice is directly attributable to the bat regulatory sequences. Heterozygous and homozygous mice for the bat Prx1 limb enhancer allele are viable, fertile, segregate in the expected Mendelian ratio, and show no readily apparent limb or craniofacial phenotypes. However, skeletal analysis of $\operatorname{Pr} x 1^{\text {BatE/BatE }}$ mice reveals that the forelimbs of these animals are about $6 \%$ longer than their wild-type littermates by the end of gestation, and this difference is statistically significant. Histological and molecular analyses indicate that bat enhancer-driven expression of $\operatorname{Prx} 1$ results in accelerated endochondral bone growth in the mutant forelimbs, caused at least in part by increased proliferation of chondrocyte precursors at the ends of the long bones. Quantitative reverse transcriptase-polymerase chain reaction (Q-RT-PCR) analysis of 17.5-day postcoitus (dpc) humerus from $\operatorname{Prx} 1^{\text {BatE }}$ homozygotes showed $\sim 1$.7-fold higher levels of Prx1 transcripts, suggesting that $\operatorname{Pr} x 1^{\text {BatE }}$ is a hypermorphic allele.

To test whether this Prxl limb enhancer is essential for limb-specific transcription, we also generated mice lacking it using a similar gene targeting strategy. To our surprise, mice homozygous for the Prxl enhancer deletion allele are viable, fertile, and do not exhibit the limb defects of Prx1 null mutants. Q-RT-PCR analysis of 17.5-dpc humerus from enhancer deletion homozygotes showed no significant difference in Prxl RNA levels in comparison to controls. Thus, although the mouse limb enhancer is 
sufficient to direct limb-specific expression of heterologous genes, it is not required for limb transcription.

These results show that divergence of a single nonessential cis-regulatory region of one gene is by itself sufficient to elicit a discrete and measurable change in limb morphogenesis. They also suggest that regulatory redundancy may be part of a general mechanism for gain of novel expression patterns among conserved essential functions. In addition to $\operatorname{Pr} x 1$, the mouse genome contains a $\operatorname{Pr} x 2$ gene, most likely a result of gene duplication. $\operatorname{Pr} x 2$ is also expressed in the mouse limb. Prx2 homozygous mutant mice are viable with normal limbs (ten Berge et al. 1998). However, Prx 1;Prx2 double-mutant mice have severely shortened limbs, suggesting that Prxl and Prx 2 interact to regulate limb length (ten Berge et al. 1998). In our Prx $1^{\text {BatE }}$ mutants, it is possible that PRX2 buffers the actions of bat-regulated PRX1 for limb development. These findings suggest that there are multiple levels of redundancy for buffering the effects of mutations on genes that regulate developmental processes.

\section{CONCLUSIONS}

The bat provides an excellent mammalian model system to explore the developmental genetics of animal diversity. The development of $C$. perspicillata as a model system has greatly facilitated research in the reproductive biology and embryology of bats. There are many morphological and physiological characteristics of developing bat embryos that show diversity relative to primary model organisms, such as the chick and mouse, beyond the limbs. For example, studying their craniofacial development would be a very exciting avenue for future research. Gene expression studies of developing bat embryos are still very limited. It would be useful to have a comprehensive analysis of gene expression in the developing limbs of bats. Such studies would likely generate candidate genes that are responsible for the generation of a wing. The reproductive biology of most bats, i.e., a relatively long gestation resulting in a single offspring and length to sexual maturity, makes the possibility of genetic studies, at best, unlikely. Thus, assaying the activity of bat sequences in the mouse with its tremendous genetic advantages can be a productive way to identify factors that lead to divergent morphologies among species.

\section{ACKNOWLEDGMENTS}

We are grateful for the kindness and assistance given to us by the many people of Trinidad who have helped us to find bat roosts, loaned us ladders, fed us wonderful food, and educated us about West Indian natural history and culture. This work was supported by National Science Foundation grant IBN 0220458 and the Ben F. Love Endowment to R.R.B.

\section{REFERENCES}

Badwaik NK, Rasweiler JJ IV, Oliveira SF. 1997. Formation of reticulated endoderm, Reichert's membrane, and amniogenesis in blastocysts of captive-bred, short-tailed fruit bats,
Carollia perspicillata. Anat Rec 247: 85-101.

Behringer RR, Eakin GS, Renfree MB. 2006. Mammalian diversity: Gametes, embryos, and reproduction. Mol Reprod Dev 18: $99-107$.

Chen C-H, Cretekos CJ, Rasweiler JJ IV, Behringer RR. 2005. Hoxd13 expression in the developing limbs of the short-tailed fruit bat, Carollia perspicillata. Evol Dev 7: 130-141.

Cretekos CJ, Rasweiler JJ IV, Behringer RR. 2001. Comparative studies on limb morphogenesis in mice and bats, a functional genetic approach towards a molecular understanding of diversity in organ formation. Reprod Fertil Dev 13: 691-695.

Cretekos CJ, Weatherbee SD, Chen C-H, Badwaik NK, Niswander L, Behringer RR, and Rasweiler JJ IV. 2005. Embryonic staging system for the short-tailed fruit bat, Carollia perspicillata, a model organism for the mammalian order Chiroptera, based upon timed pregnancies in captive-bred animals. Dev Dyn 233: 721-738.

Cretekos CJ, Deng JM, Green ED, NISC Comparative Sequencing Program, Rasweiler JJ IV, Behringer RR. 2007. Isolation, genomic structure and developmental expression of Fgf8 in the short-tailed fruit bat, Carollia perspicillata. Int J Dev Biol 51: 333-338.

Cretekos CJ., Wang Y, Green ED, NISC Comparative Sequencing Program, Martin JF, Rasweiler JJ IV, Behringer RR. 2008. Regulatory divergence modifies forelimb length in mammals. Genes Dev 22: 141-151.

Cserjesi P, Lilly B, Bryson L, Wang Y, Sassoon DA, Olson EN. 1992. MHox: A mesodermally restricted homeodomain protein that binds an essential site in the muscle creatine kinase enhancer. Development 115: 1087-1101.

Giannini N, Goswami A, Sanchez-Villagra MR. 2006. Development of integumentary structures in Rousettus amplexicaudatus (Mammalia: Chiroptera: Pteropodidae) during late-embryonic and fetal stages. J Mammalogy 87: 993-1001.

Hockman D, Mason MK, Cretekos CJ, Behringer RR, Jacobs DS, Illing N. 2008. A second wave of Sonic Hedgehog expression during the development of the bat limb. Proc Natl Acad Sci 105: $16982-16987$.

Hockman D, Mason MK, Jacobs DS, Illing N. 2009. The role of early development in mammalian limb diversification: A descriptive comparison of early limb development between the Natal long-fingered bat (Miniopterus natalensis) and the mouse (Mus musculus). Dev Dyn 238: 965-979.

Lu MF, Cheng HT, Lacy AR, Kern MJ, Argao EA, Potter SS, Olson EN, Martin JF. 1999. Paired-related homeobox genes cooperate in handplate and hindlimb zeugopod morphogenesis. Dev Biol 205: 145-157.

Martin JF, Olson EN. 2000. Identification of a prxl limb enhancer. Genesis 2: 225-229.

Martin JF, Bradley A, Olson EN. 1995. The paired-like homeo box gene MHox is required for early events of skeletogenesis in multiple lineages. Genes Dev 9: 1237-1249.

Nolte MJ, Hockman D, Cretekos CJ, Behringer RR, Rasweiler JJ IV. 2009. Embryonic staging system for the black mastiff bat, Molossus rufus (Molossidae), correlated with structure-function relationships in the adult. Anat Rec 292: 155-168.

Rasweiler JJ IV, Oliveira SF, Badwaik S.F. 2000. An ultrastructural study of interstitial implantation in captive-bred, shorttailed fruit bats, Carollia perspicillata: Trophoblastic adhesion and penetration of the uterine epithelium. Anat Embryol 205: 371-391.

Rasweiler JJ IV, Cretekos CJ, Behringer RR. 2009. The shorttailed fruit bat Carollia perspicillata: A model for studies in reproduction and development. In Emerging model organisms: A laboratory manual, vol. 1, pp. 519-555. Cold Spring Harbor Laboratory Press, Cold Spring Harbor, NY.

Ray R, Capecchi M. 2008. An examination of the chiropteran HoxD locus from an evolutionary perspective. Evol Dev 10: 657-670.

Sears KE, Behringer RR, Rasweiler JJ IV, Niswander LA. 2006. The development of bat flight, morphological and molecular evolution of bat wing digits. Proc Natl Acad Sci 103: 65816586.

ten Berge D, Brouwer A, Korving J, Martin JF, Meijlink F. 1998. 
Prx 1 and Prx2 in skeletogenesis: Roles in the craniofacial region, inner ear and limbs. Development 125: 3831-3842.

Tokita M. 2006. Normal embryonic development of the Japanese pipistrelle, Pipistrellus abramus. Zoology 109: 137-147.
Weatherbee SD, Behringer RR, Rasweiler JJ IV, Niswander LA. 2006. Interdigital webbing retention in bat wings illustrates genetic changes underlying amniote limb diversification. Proc Natl Acad Sci 103: 15103-15107. 


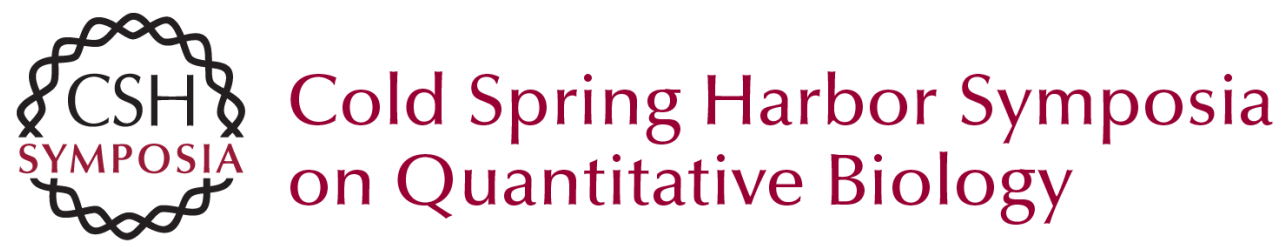

\title{
Genetic Regulation of Mammalian Diversity
}

\author{
R.R. Behringer, J.J. Rasweiler IV, C.-H. Chen, et al.
}

Cold Spring Harb Symp Quant Biol 2009 74: 297-302 originally published online December 22, 2009 Access the most recent version at doi:10.1101/sqb.2009.74.035

References This article cites 22 articles, 7 of which can be accessed free at: http://symposium.cshlp.org/content/74/297.full.html\#ref-list-1

License

Email Alerting Receive free email alerts when new articles cite this article - sign up in the box at the Service top right corner of the article or click here. 